\title{
Drinking Hydrogen-Rich Water Alleviates Chemotherapy-Induced Neuropathic Pain Through the Regulation of Gut Microbiota
}

This article was published in the following Dove Press journal: Journal of Pain Research

\author{
Naqi Lian ${ }^{1,2, *}$ \\ Mengxi Shen ${ }^{1,2, *}$ \\ Kai Zhang ${ }^{1,2, *}$ \\ Jiacheng Pan ${ }^{1,2}$ \\ Yi Jiang ${ }^{1,2}$ \\ Yang $Y u$ iD $^{1,2}$ \\ Yonghao $\mathrm{Yu}^{\mathrm{l}, 2}$ \\ 'Department of Anesthesiology, Tianjin \\ Medical University General Hospital, \\ Tianjin, 300052, People's Republic of \\ China; ${ }^{2}$ Tianjin Institute of \\ Anesthesiology, Tianjin, People's Republic \\ of China
}

*These authors contributed equally to this work
Correspondence: Yang Yu; Yonghao Yu Department of Anesthesiology, Tianjin Medical University General Hospital, 154 Anshan Road, Tianjin, 300052, People's Republic of China

Email sevenyu1987@outlook.com; yuyonghao@126.com
Introduction: Chemotherapy-induced neuropathic pain (CINP) is one of the most common complications of chemotherapeutic drugs which limits the dose and duration of potentially life-saving anticancer treatment and compromises the quality of life of patients. Our previous studies have reported that molecular hydrogen $\left(\mathrm{H}_{2}\right)$ can be used to prevent and treat various diseases. But the underlying mechanism remains unclear. The aim of the present study was to explore the effects of hydrogen-rich water on gut microbiota in CINP.

Methods: All C57BL/6J mice were divided into 4 groups: The group fed with normal drinking water and injected with saline $\left(\mathrm{H}_{2} \mathrm{O}+\right.$ Saline $)$, the group fed with normal drinking water and injected with oxaliplatin $\left(\mathrm{H}_{2} \mathrm{O}+\mathrm{OXA}\right)$, the group fed with hydrogen-rich water and injected with saline (HW + Saline), and the group fed with hydrogen-rich water and injected with oxaliplatin (HW + OXA). The mechanical paw withdrawal threshold of the mice was tested on days $0,5,10,15$ and 20 after hydrogen-rich water treatment. On day 20, feces of mice from different groups were collected for microbial community diversity and structure analysis. The levels of inflammatory cytokines (TNF- $\alpha$ and IL-6), oxidative stress factors $\left(\mathrm{OH}^{-}\right.$and $\left.\mathrm{ONOO}^{-}\right)$, lipopolysaccharide (LPS) and Toll-like receptor 4 (TLR4) were detected in dorsal root ganglia (DRG), L4-6 spinal cord segments and serum by enzymelinked immunosorbent assay. The expression of TLR4 in DRG and spinal cords was determined by Western blot.

Results: The results illustrated that hydrogen-rich water could alleviate oxaliplatin-induced hyperalgesia, reduce the microbial diversity and alter the structure of gut microbiota, reverse the imbalance of inflammatory cytokines and oxidative stress, and decrease the expression of LPS and TLR4.

Conclusion: Hydrogen-rich water may alleviate CINP by affecting the diversity and structure of the gut microbiota, and then the LPS-TLR4 pathway, which provides a direction for further research.

Keywords: hydrogen-rich water, gut microbiota, CINP, LPS-TLR4 pathway

\section{Introduction}

Chemotherapy is an important method for the treatment of malignant tumors. ${ }^{1}$ However, chemotherapeutic drugs, such as oxaliplatin, often cause several adverse reactions. Chemotherapy-induced neuropathic pain (CINP) is one of the most common complications caused by treatment with chemotherapeutic drugs such as oxaliplatin. Chemotherapeutic drug-induced neuropathic pain in the extremities limits the dosing and duration of potentially life-saving anticancer treatment, thus impairing the quality of life of patients. ${ }^{2}$ The main clinical manifestations are 
continuous spontaneous pain, intermittent burning pain, severe hyperalgesia and hypersensitivity of the entire or part of the limbs following the administration of chemotherapy drugs. ${ }^{3}$ Since the incidence rate of neuropathic pain is as high as $70 \%$ and there is no effective treatment, patients experiencing severe pain often reduce or even discontinue the chemotherapy drugs. This results in failure of timely administration of new chemotherapy cycles, a decrease in the chemotherapy dose and even treatment interruption, thus seriously affecting the quality of life and prognosis of patients with malignant tumors. ${ }^{4}$

A variety of microbial communities located in the intestinal tract are collectively referred to as gut microbiota. ${ }^{5}$ The gut microbiota provides important benefits to its hosts and plays a crucial role in maintaining the homeostasis of the gastrointestinal tract. The natural gut microbiota gets along with the mucosal epithelium and performs a variety of physiological functions, such as regulating intestinal barrier integrity, maintaining substance metabolism, and regulating immune and inflammatory responses. ${ }^{6,7}$ Markers of microbial stability, such as abundance and diversity, are often used as indicators of intestinal soundness, due to their negative correlation with chronic diseases and metabolic dysfunction. ${ }^{8,9}$ It has been pointed out that the application of chemotherapeutic drugs only caused neuropathic pain in a specific pathogen-free mouse model, with no chemotherapy-induced pain observed in germ-free mice. ${ }^{5}$

Molecular hydrogen $\left(\mathrm{H}_{2}\right)$, which is widely distributed in nature, is the smallest, lightest and richest element in the universe, ${ }^{10}$ and has antioxidant, anti-inflammatory and anti-apoptotic functions. ${ }^{11}$ As a potential oxidation inhibitor, hydrogen gas inhalation or hydrogen-rich water drinking been have been extensively used in medical applications without side effects. ${ }^{12,13}$ Our previous studies have reported that $2 \%$ hydrogen gas inhalation and hydrogen-rich water drinking provided a protective effect against various diseases, including ischemia-reperfusion injury, sepsis, multiple organ dysfunction syndrome, type II diabetes and neurodegenerative diseases in animal or cell models. ${ }^{11,14-17}$ A 2016 report described an interesting case, in which $70 \%$ of gastrointestinal microbial species exhibited a genetic capacity to metabolize $\mathrm{H}_{2}$, suggesting that $\mathrm{H}_{2}$ may affect intestinal microbial activity, population or community. ${ }^{18}$ Given the protective effects of $\mathrm{H}_{2}$ on chronic diseases and metabolic dysfunction, hydrogenrich water drinking therapy may affect the abundance and diversity of the gut microbiota.
Toll-like receptors (TLRs) are an important class of transmembrane protein molecules involved in nonspecific immunity (native immunity). They can specifically recognize various exogenous and endogenous danger signals, thereby activating the innate immune system to maintain the survival of the host. They are also considered bridges connecting non-specific and specific immunity. ${ }^{19,20}$ TLR4 is the first toll-like receptor-related protein identified in humans, and is expressed on the surface of non-specific immune cells, such as macrophages and neutrophils, as well as on T and B lymphocytes, which mediate specific immune responses. TLR4 can specifically recognize the endotoxin and lipopolysaccharide (LPS) released by bacteria. $^{21}$ The combination of LPS and TLR4 can trigger a series of downstream events, eventually leading to the release of a large number of inflammatory mediators (such as TNF- $\alpha$ and IL-6). In recent years, it has been shown that the gut microbiota may affect the development of chemotherapy-induced pain through the LPS-TLR4 pathway. ${ }^{5}$ Therefore, in the present study, it was hypothesized that hydrogen-rich drinking water therapy could inhibit the LPS-TLR4 pathway and its downstream response by regulating the abundance and variability of the gut microbiota, thereby exerting therapeutic effects on CINP.

\section{Materials and Methods}

\section{Animals}

This experiment protocol was authorized by the Institutional Animal Care and Use Committee of the Tianjin Medical University (approval no. IRB2019-DW -07) and performed according to the guidelines of the National Institutes of Health for the care and use of experimental animals. Female C57BL/6J mice aged 8 weeks and weighing 20-25 g each were purchased from the Laboratory Animal Center of the Military Medical Science Academy (Beijing, China). Animals were housed in cages at room temperature $\left(20-22^{\circ} \mathrm{C}\right)$ under a regular $12 / 12 \mathrm{~h}$ light/dark cycle fed with food and water ad libitum.

\section{Experimental Protocols}

Figure 1 shows the experimental plan. The mice were randomly separated into 4 groups: The mice fed with normal drinking water and injected with saline $\left(\mathrm{H}_{2} \mathrm{O}+\right.$ Saline group), the mice fed with normal drinking water and injected with oxaliplatin $\left(\mathrm{H}_{2} \mathrm{O}+\mathrm{OXA}\right.$ group $)$, the 


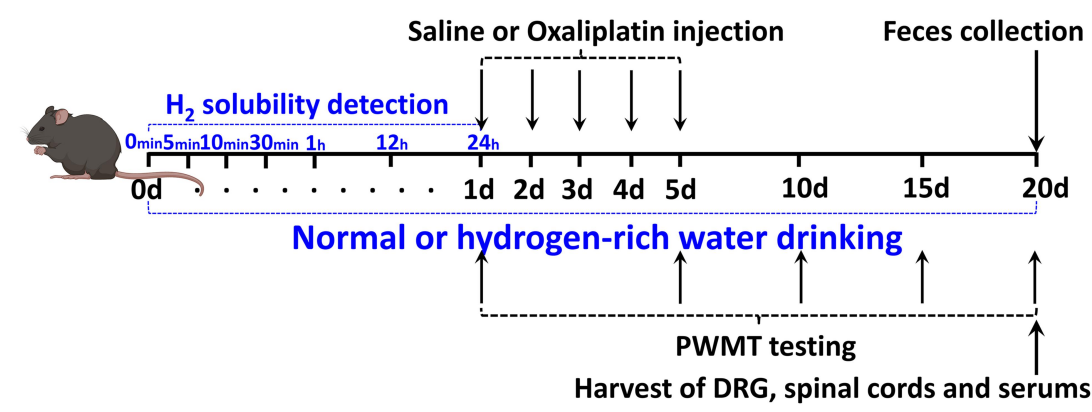

Figure I Experimental design. WT female C57BL/6] mice aged 8 weeks and weighing 20-25 g were subjected to saline or oxaliplatin injection. Hydrogen-rich or normal drinking water was used for the entire experiment. One day before the OXA or saline injection (on day 0 ), the solubility of $\mathrm{H}_{2}$ in normal or hydrogen-rich water was detected at 0, 5, 10 and $30 \mathrm{~min}$, and I, I 2 and $24 \mathrm{~h}$ after blowing the hydrogen gas into the drinking water. PWMT was carried out I, 5, I0, I5 and 20 days after the saline or oxaliplatin injection. The feces of different groups were obtained for tests following PWMT test. The DRG and spinal cords of L4-6 segments and serum of different groups were obtained for tests following feces collection on day 20. Different groups of DRG, spinal cords and serum were used for ELISA, and DRG and spinal cords were used for Western blot analysis, as mentioned in the Materials and Methods section.

Abbreviations: WT, wild-type; PWMT, paw withdrawal mechanical threshold; DRG, dorsal root ganglia.

mice fed with hydrogen-rich water and injected with saline (HW + Saline group) and the mice fed with hydrogen-rich water and injected with oxaliplatin (HW + OXA group). Oxaliplatin was injected at a concentration of $0.2 \mathrm{mg} / \mathrm{mL}$. Mice in the $\mathrm{H}_{2} \mathrm{O}+$ OXA and $\mathrm{HW}+\mathrm{OXA}$ groups were injected intraperitoneally with oxaliplatin solution at a dose of $3 \mathrm{mg} / \mathrm{kg}$ each time for 5 consecutive days (from days 1 to 5), and the mice in the $\mathrm{H}_{2} \mathrm{O}+$ Saline and HW + Saline groups were injected intraperitoneally with the same dose of normal saline for 5 days; the hydrogen-rich water was treated during the whole experiment. One day before the OXA or Saline injection (on day 0 ), the solubility of $\mathrm{H}_{2}$ in normal or hydrogenrich water was detected at $0,5,10$ and $30 \mathrm{~min}$, and 1,12 and $24 \mathrm{~h}$ after blowing the hydrogen gas into the drinking water. Mice were tested for mechanical paw withdrawal mechanical threshold (PWMT) at 1, 5, 10, 15 and 20 days after treatment with hydrogen-rich water. Next, the feces of mice from all groups were gathered for microbial community diversity analysis on day 20 . The mice were anesthetized by an injection of $2 \%$ sodium pentobarbital (diluted to $50 \mathrm{mg} / \mathrm{kg}$ and dissolved in saline) into the abdominal cavity and then sacrificed by cervical dislocation following feces collection to obtain their DRG, L4-L6 segments of their spinal cords and serum on day 20. Acquired specimens from 5 mice from each group were used for inflammatory cytokine (TNF- $\alpha$ and IL-6), oxidative stress indicator $\left(\mathrm{OH}^{-}\right.$and $\left.\mathrm{ONOO}^{-}\right)$, LPS and TLR4 determination by enzyme-linked immunosorbent assay (ELISA). DRG and spinal cords from 5 mice per group were used to measure TLR4 by Western blot analysis.

\section{Preparation of Hydrogen-Rich Water}

The normal drinking water was put in a drinking bottle with an inlet and an outlet, and a hydrogen gas was generated by a GCH-300 high-purity hydrogen generator (Tianjin Tongpu Analytical Instrument Technology Co., Ltd.), and hydrogen gas was blown into the drinking water at a flow rate of $400 \mathrm{~mL} / \mathrm{min}$ for $10 \mathrm{~min}$ to prepare hydrogen-rich water $\left[\mathrm{H}_{2}\right.$ solubility, $>1,000$ part per billion $\left.(\mathrm{PPb})\right]$. After $10 \mathrm{~min}$, the gas flow rate was changed to $100 \mathrm{~mL} / \mathrm{min}$; blowing into the drinking water was continued to maintain the solubility of hydrogen molecules in the hydrogen-rich water (800$1,000 \mathrm{PPb}$ ) and discharge the exhaust gas through the outlet of the drinking bottle to the exhaust gas recovery device. The drinking water bottle was placed in a cage for the mice to drink from for $24 \mathrm{~h}$. Hydrogen-rich water needed daily replacement to ensure hydrogen concentration and cleanliness of drinking water.

\section{$\mathrm{H}_{2}$ Solubility Measurement}

The solubility of $\mathrm{H}_{2}$ in normal or hydrogen-rich water was detected at 0, 5, 10 and $30 \mathrm{~min}$, and 1, 12 and $24 \mathrm{~h}$ after blowing the hydrogen gas into the drinking water by using a dissolved hydrogen tester (ENH-1000 Molecular Hydrogen $\mathrm{H}_{2}$ Meter, Trustlex, Japan), according to the manufacturer's instructions. The $\mathrm{H}_{2}$ solubility was measured three times at each time point.

\section{Preparation of CINP Animal Model}

Oxaliplatin was dissolved in physiological saline to prepare a $0.2 \mathrm{mg} / \mathrm{mL}$ suspension. Mice in the $\mathrm{H}_{2} \mathrm{O}+\mathrm{OXA}$ and HW + OXA groups were injected intraperitoneally with oxaliplatin solution at a dose of $3 \mathrm{mg} / \mathrm{kg}$ each time; 
they were continuously injected for 5 days to prepare a CINP animal model. Mice in the $\mathrm{H}_{2} \mathrm{O}+$ Saline and $\mathrm{HW}+$ Saline groups were injected intraperitoneally with the same dose of normal saline for 5 days. The experiment was performed as previously described. ${ }^{5}$

\section{Behavioral Test}

All animals adapted to the test environment for 3 days before measuring the pain threshold. The electronic Von Frey filaments (BSEVF3; Harward Apparatus Co., USA) were used to detect the mechanical stimulation, and the figures were recorded as PWMT. PWMT was measured as previously described. $^{22}$

\section{Feces Sample Collection and Preparation}

The mice defecated by a forced method or by abdominal massage, and the feces samples were collected directly in a sterile tube. Samples collected from the same mouse were evenly mixed, dispensed into $2 \mathrm{~mL}$ imported sterile cryotubes, weighed and recorded, and a sufficient quantity was collected for multiple backups of each sample. The specimens were frozen using liquid nitrogen for $15 \mathrm{~min}$, and were then kept at $-80^{\circ} \mathrm{C}$ in sterile cryotubes as previously described. ${ }^{23}$ For the analysis of microbial community diversity, the total genomic DNA in the samples was drawn with the CTAB/SDS method. The 16S/18S rRNA gene was amplified using a specific primer with a barcode. An equal volume of $1 \mathrm{X}$ loading buffer (containing SYB green) was admixed with the PCR product and electrophoresed on a $2 \%$ agarose gel for detection. Specimens with a bright main strip between 400-450 bp were selected for further tests. The PCR products were mixed in equal proportions. The PCR product was then purified using the GeneJET Gel Extraction Kit (Thermo Fisher Scientific, Inc.). Following the manufacturer's instructions, a sequencing library was produced by the NEB $\mathrm{Next}^{\circledR}$ UltraTM DNA Illumina Library Preparation Kit (NEB, USA) with an index code added. The library quality was evaluated on a Qubit@2.0 fluorometer (Thermo Fisher Scientific, Inc.) and an Agilent Bioanalyzer 2100 system. Finally, the library was sequenced on the Illumina MiSeq platform and paired end reads of 250/300 bp were produced.

\section{Analysis of Microbial Community Diversity} Operational Classification Unit (OTU) is the same marker artificially set for a taxonomic unit (strain, genus, species, population, etc.) to facilitate analysis in phylogeny or population genetics studies. Sequence analysis was performed by UPARSE software package using the UPARSE-OTUref and UPARSE-OTU algorithms. Internal Perl scripts were applied to analyze alpha (withinsample) and beta (among-sample) diversity. The sequences with similarity $\geq 97 \%$ were assigned to the same OTUs. A representative sequence was selected for each OTU and the RDP classifier was used to label the classification information for each representative sequence. To calculate a-diversity, the OTU table was confirmed and three indicators were calculated: The abundance of species was evaluated by Chaol estimates; the number of unique OTUs discovered in each specimen and the Shannon and Simpson indices were estimated by species observation. Rarefaction curves were created on account of these three indicators. $^{23-25}$

\section{ELISA}

DRG, spinal cords and serum were obtained following centrifugation at $3,000 \times \mathrm{g}$ for $15 \mathrm{~min}$ at $4{ }^{\circ} \mathrm{C}$ to estimate the expression of inflammatory cytokines (TNF- $\alpha$ and IL-6), oxidative stress indicators $\left(\mathrm{OH}^{-}\right.$and $\left.\mathrm{ONOO}^{-}\right)$, LPS and TLR4 by commercially available ELISA kits (mouse TNF- $\alpha$, IL-6, LPS and TLR4 ELISA kits from SenBeiJia Biological Technology Co., Ltd.; $\mathrm{OH}^{-}$and $\mathrm{ONOO}^{-}$kits from Jianglai Biological Technology Co., Ltd.) using a microplate reader, following the manufacturers' instructions. All samples were run in duplicate.

\section{Western Blot Analysis}

Western blot analysis was performed to confirm the expression of TLR4 in the DRG and spinal cords of mice. The samples were separated using SDS-PAGE by 8\% double TIS polyacrylamide gel (cat no. M00655; GenScript Biotech Corporation) and transferred to a nitrocellulose membrane (Bio-Rad Laboratories, Inc.). The membrane was incubated in 5\% skimmed milk powder and detected using the following antibodies: $\beta$-Actin (cat no. 4970T; dilution, 1:1,000; Cell Signaling Technology, Inc.) and TLR4 (cat no. 14358S; dilution, 1:1,000; Cell Signaling Technology, Inc.). Following rinsing at $4^{\circ} \mathrm{C}$ overnight, goat anti-rabbit antibody (cat no. abs20002ss; dilution, 1:8,000; Absin Bioscience Inc.) was added, followed by incubation at $37^{\circ} \mathrm{C}$ for $1 \mathrm{~h}$. The nitrocellulose membrane was washed in TBST and dripped with ECL chemiluminescent liquid (cat no. 34577; Thermo Fisher Scientific, Inc.), and then scanned and photographed by Bio-Rad image analysis system (BioRad Laboratories, Inc.). The expression level of the target 
protein was reflected by the ratio of the integral optical density of the target line in relation to the $\beta$-actin.

\section{Statistical Analysis}

Microbial community differences were analyzed using analysis of similarities, multi-response permutation procedures and Bray-Curtis differential distance matrix. Linear discriminant analysis Effect Size was used to quantitatively analyze biomarkers in different groups. Two-way analysis of variance was performed to analyze the differences between groups in all comparisons. Statistical analysis was performed by the GraphPad Prism software (version 8.0) and SPSS statistical software (version 21.0). $\mathrm{P}<0.05$ was considered to indicate a statistically significant difference, as determined by two-tail test. All data are expressed as the mean \pm SD.

\section{Results}

\section{Solubility of $\mathrm{H}_{2}$ in Normal and Hydrogen-Rich Water at Different Time Points}

The solubility of $\mathrm{H}_{2}$ in normal or hydrogen-rich water was detected at 0,10 and $30 \mathrm{~min}$, and 1,12 and $24 \mathrm{~h}$ after blowing hydrogen gas or nothing into the drinking water. The results showed that there was no $\mathrm{H}_{2}$ dissolved in normal drinking water, the $\mathrm{H}_{2}$ solubility in normal water was $0 \mathrm{ppb}$ at all time points. In the first $10 \mathrm{~min}$, the blow rate of $\mathrm{H}_{2}$ was $400 \mathrm{~mL} / \mathrm{min}$ and the $\mathrm{H}_{2}$ solubility increased quickly with time, peaking at $1,200 \mathrm{ppb}$. The blow rate of $\mathrm{H}_{2}$ was then changed to $100 \mathrm{~mL} / \mathrm{min}$, and the solubility of $\mathrm{H}_{2}$ decreased slightly and maintained at $900-1,000 \mathrm{ppb}$ until the $24 \mathrm{~h}$ time point (Figure 2).

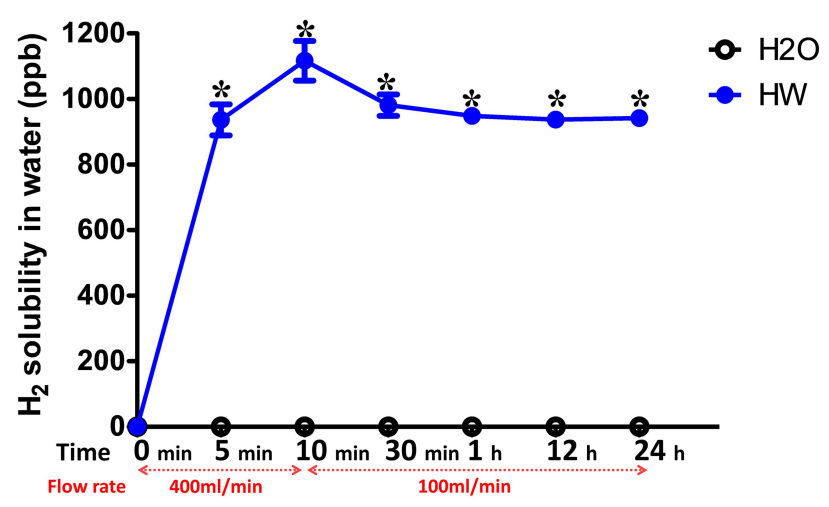

Figure 2 Solubility of $\mathrm{H}_{2}$ in normal and hydrogen-rich water at different time points. The solubility of $\mathrm{H}_{2}$ in normal or hydrogen-rich water was detected at 0,10 and $30 \mathrm{~min}$, and $\mathrm{I}, \mathrm{I} 2$ and $24 \mathrm{~h}$ after blowing the hydrogen gas into the drinking water. The flow rate of $\mathrm{H}_{2}$ was $400 \mathrm{~mL} / \mathrm{min}$ at the first 10 min and $100 \mathrm{~mL} / \mathrm{min}$ from 10 min to $24 \mathrm{~h}$. The $\mathrm{H}_{2}$ solubility was measured three times at each time point. $\mathrm{H}_{2}$, molecular hydrogen. $* \mathrm{P}<0.05$ vs $\mathrm{H}_{2} \mathrm{O}$ group.

\section{Hydrogen-Rich Water Treatment Significantly Alleviates Mechanical Hyperalgesia in Mice with Oxaliplatin-Induced Hyperalgesia}

Mice were exposed to normal drinking water or hydrogenrich water with an injection of saline or oxaliplatin. As shown in Figure 3, there was no significant difference in the baseline of mechanical thresholds between the HW + Saline and $\mathrm{H}_{2} \mathrm{O}+$ Saline groups $(\mathrm{P}>0.05$, $\mathrm{HW}+$ Saline vs $\mathrm{H}_{2} \mathrm{O}+$ Saline). In addition, as compared with the $\mathrm{H}_{2} \mathrm{O}+$ OXA group, hydrogen-rich water significantly improved the mechanical hyperalgesia of CINP mice at 10 days $\left(\mathrm{P}<0.05\right.$, HW + OXA vs $\mathrm{H}_{2} \mathrm{O}+$ OXA). Based on these data, hydrogen-rich water was found to exert a significant protective effect on mice with oxaliplatin-induced CINP.

\section{Hydrogen-Rich Water Reduces the Diversity and Alters the Structure of Gut Microbiota in Mice}

The diversity and structure of gut microbiota was detected by amplifying and sequencing the $16 \mathrm{~S}$ rDNA genes. In order to prove that the results obtained from the samples represented the true state of the microorganisms, Good's coverage index, was used to determine the coverage of each sample library (Figure 4A), and all sets of data were close to 1 ; this meant the sequencing results represented the true state of the microorganisms in the sample. The diversity of gut microbiota in the four groups of mice is presented as community diversity (Figure 4B and C). As compared with the mice drinking ordinary water, hydrogen-rich water significantly increased the Simpson index and decreased the Shannon index $\left(\mathrm{P}<0.05\right.$, HW + OXA vs $\mathrm{H}_{2} \mathrm{O}+\mathrm{OXA}$; $\mathrm{P}<0.05$, HW + Saline vs $\mathrm{H}_{2} \mathrm{O}+$ Saline); however, there were no statistical differences in the community diversity of mice that drunk the same water $\left(\mathrm{P}>0.05, \mathrm{H}_{2} \mathrm{O}+\right.$ Saline vs $\mathrm{H}_{2} \mathrm{O}+$ OXA; P>0.05, HW + Saline vs HW + OXA).

Specific to the results of species classification, the gut microbiota structure in four groups of mice was presented as the relative abundance of gut microbiota at the phylum, family and genus levels. At the phylum level, hydrogen-rich water treatment raised the relative abundance of Firmicutes and Tenericutes, and reduced the relative abundance of Bacteroidetes $\left(\mathrm{P}<0.05\right.$, HW + Saline vs $\mathrm{H}_{2} \mathrm{O}+$ Saline; $\mathrm{P}<0.05$, HW + OXA vs $\mathrm{H}_{2} \mathrm{O}+\mathrm{OXA}$ ) (Figure 5A and Table S1). At the family level, the levels of Lachnospiraceae, Lactobacillaceae and Ruminococcaceae were higher in the feces of mice treated with hydrogen-rich water $(\mathrm{P}<0.05$, HW 
A

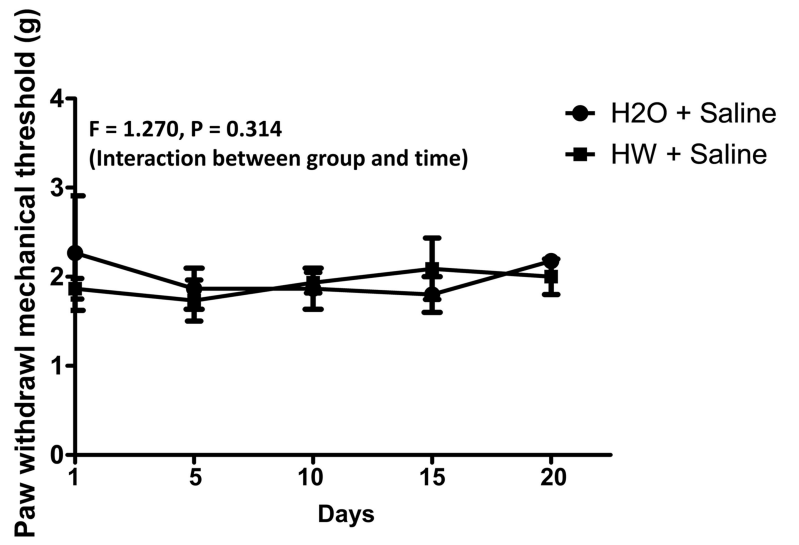

B

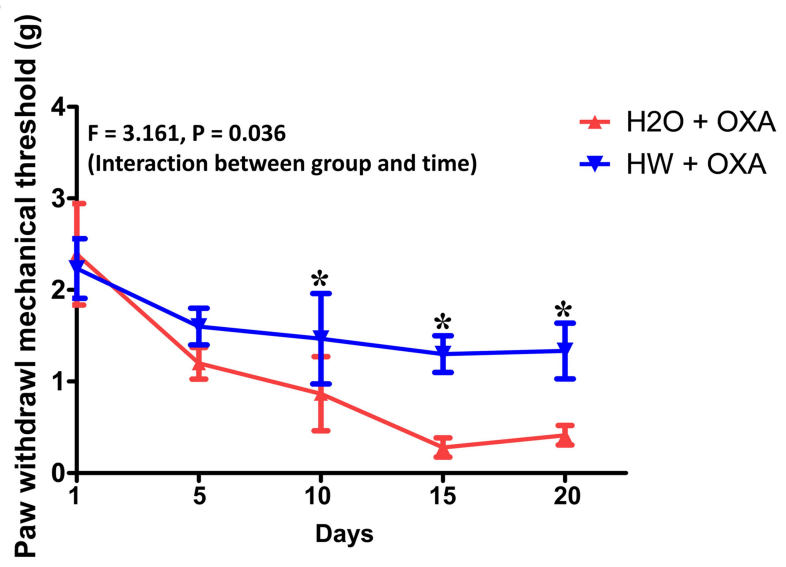

Figure 3 Effects of hydrogen-rich water on paw withdrawal mechanical threshold in CINP mice. Mice were treated with hydrogen-rich or normal drinking water and injected with saline or oxaliplatin for the duration of the experiment. Values are presented as PWMT between the $(\mathbf{A}) \mathrm{H}_{2} \mathrm{O}+\mathrm{Saline}$ and $\mathrm{HW}+\mathrm{Saline}$ and $(\mathbf{B}) \mathrm{H} \mathrm{O}_{2} \mathrm{O}+\mathrm{OXA}$ and $\mathrm{HW}+\mathrm{OXA}$ groups ( $\mathrm{n}=10$ mice per group). $* \mathrm{P}<0.05$ vs $\mathrm{H}_{2} \mathrm{O}+\mathrm{OXA}$ group.

Abbreviations: CINP, chemotherapy-induced neuropathic pain; PWMT, paw withdrawal mechanical threshold.

A

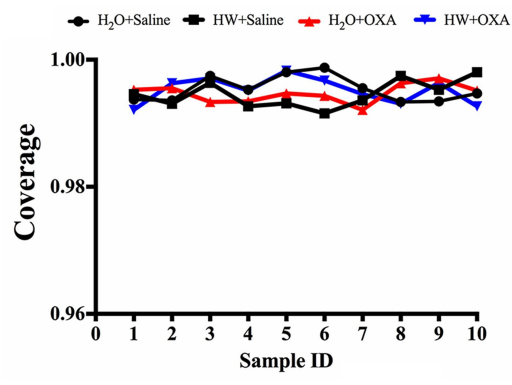

B

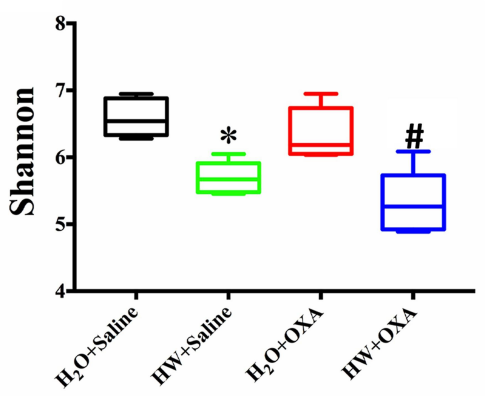

C

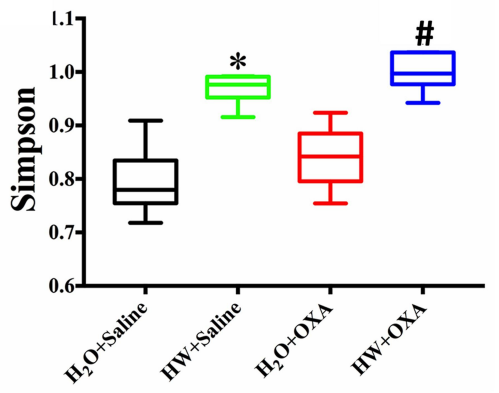

Figure 4 Effects of hydrogen-rich water on richness and $\alpha$-diversity of microbiota communities in the feces of CINP mice. Fecal samples were obtained on day 20 from mice fed with hydrogen-rich or normal drinking water and injected with saline or oxaliplatin. The plotted data are presented as the mean \pm SD of the (A) coverage, (B) Shannon and (C) Simpson indices. ${ }^{*} \mathrm{P}<0.05$ vs $\mathrm{H}_{2} \mathrm{O}+$ Saline; ${ }^{*} \mathrm{P}<0.05$ vs $\mathrm{H}_{2} \mathrm{O}+\mathrm{OXA}$ ( $\mathrm{n}=6$ mice per group).

Abbreviations: CINP, chemotherapy-induced neuropathic pain.

A

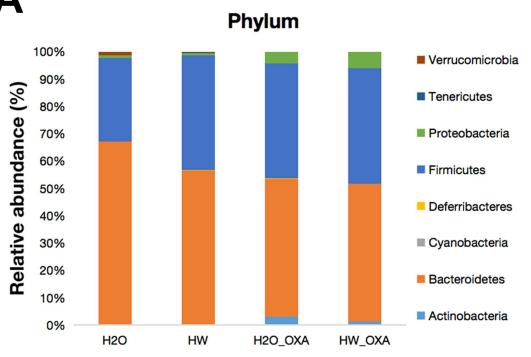

B

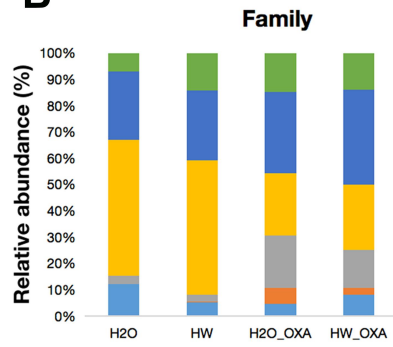

C

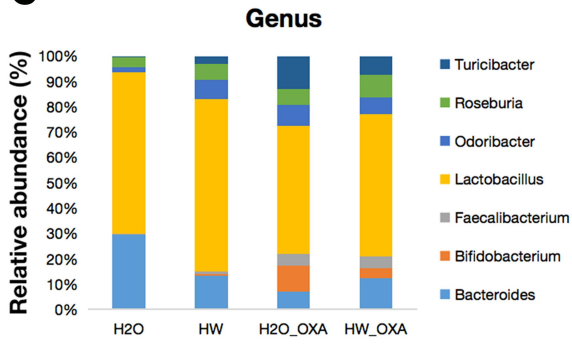

Figure 5 Effects of hydrogen-rich water on species composition and changes of microbiota communities in the feces of CINP mice. Fecal samples were obtained on day 20 from mice fed with hydrogen-rich or normal drinking water and injected with saline or oxaliplatin. (A-C) Relative abundance of intestinal microbiota at the (A) phylum, (B) family and (C) genus levels in different groups ( $n=6$ mice per group).

+ Saline vs $\mathrm{H}_{2} \mathrm{O}+$ Saline; $\mathrm{P}<0.05$, $\mathrm{HW}+$ OXA vs $\mathrm{H}_{2} \mathrm{O}+$ OXA), while Bifidobacteriaceae and Erysipelotrichaceae were only decreased in the HW + OXA group, as compared with the $\mathrm{H}_{2} \mathrm{O}+$ OXA group $\left(\mathrm{P}<0.05\right.$, $\mathrm{HW}+$ OXA vs $\mathrm{H}_{2} \mathrm{O}+$
OXA) (Figure 5B and Table S2). At the genus level, hydrogen-rich water increased the levels of Faecalibacterium, Lactobacillus and Roseburia in the feces of mice $(\mathrm{P}<0.05$, $\mathrm{HW}+$ Saline vs $\mathrm{H}_{2} \mathrm{O}+$ Saline; $\mathrm{P}<0.05$, $\mathrm{HW}+\mathrm{OXA}_{\text {vs }} \mathrm{H}_{2} \mathrm{O}+$ 
OXA). As compared to that in the $\mathrm{H}_{2} \mathrm{O}+\mathrm{OXA}$ group, the level of Bifidobacterium was significantly reduced in the $\mathrm{HW}$ + OXA group $\left(\mathrm{P}<0.05\right.$, HW + OXA vs $\left.\mathrm{H}_{2} \mathrm{O}+\mathrm{OXA}\right)$ (Figure $5 \mathrm{C}$ and Table $\mathrm{S} 3$ ). These data indicated that hydrogen-rich water could reduce community diversity and alter community structure to attenuate hyperalgesia.

\section{Hydrogen-Rich Water Reverses the} Imbalance of Inflammatory Condition and Oxidative Stress Caused by Oxaliplatin

Following treatment with oxaliplatin, the expression of inflammatory cytokines TNF- $\alpha$ and IL-6 was significantly

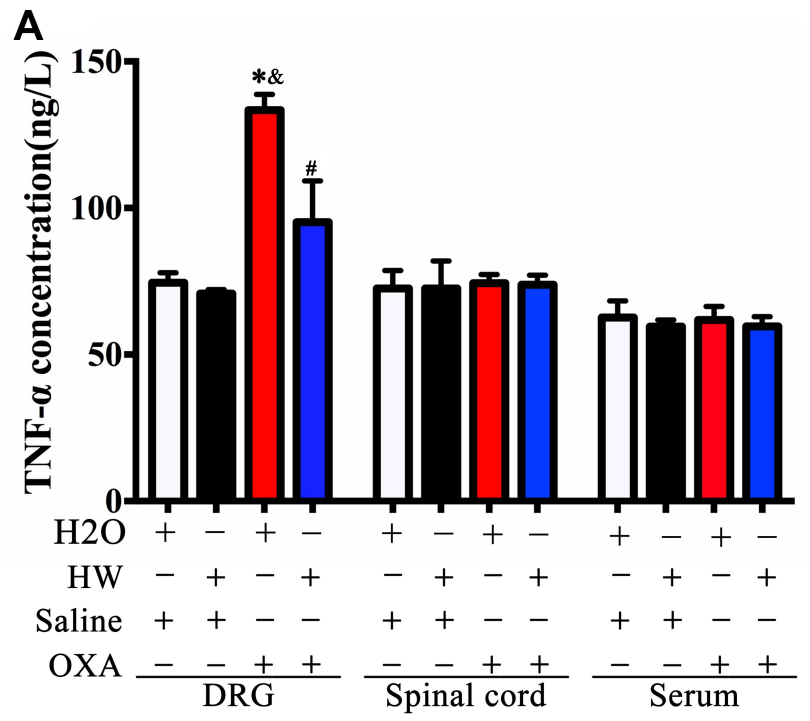

C

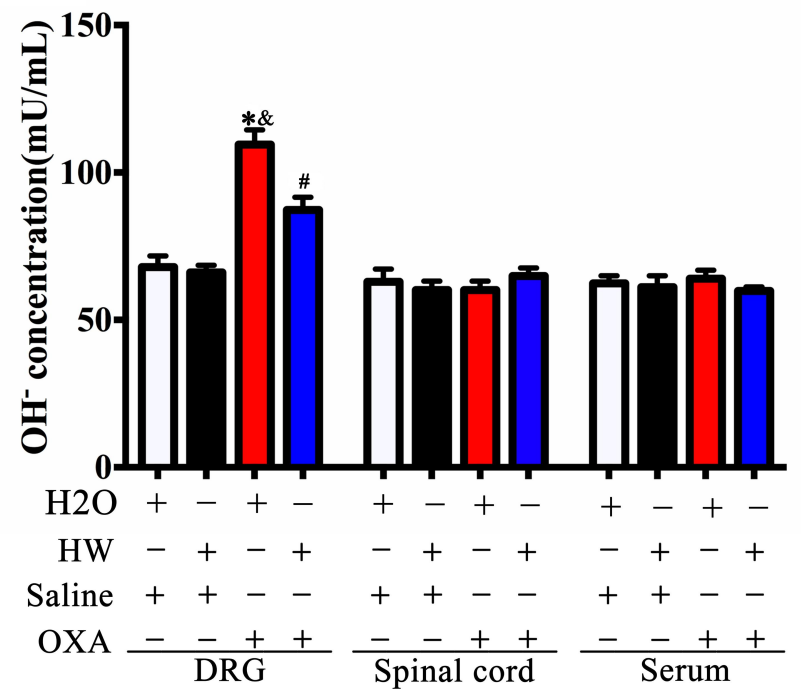

increased $\left(\mathrm{P}<0.05, \mathrm{H}_{2} \mathrm{O}+\right.$ OXA vs $\mathrm{H}_{2} \mathrm{O}+$ Saline; $\mathrm{P}<0.05$, HW + OXA vs HW + Saline). However, hydrogen-rich water could reduce the expression of TNF- $\alpha$ and IL-6 in CINP mice ( $\mathrm{P}<0.05$, HW + OXA vs $\mathrm{H}_{2} \mathrm{O}+$ OXA). Among these three types of samples, DRG, spinal cords and serum, differences in the levels of TNF- $\alpha$ and IL- 6 were only observed in DRG (Figure 6A and B).

The findings were similar for oxidative stress indicators. As compared with the saline injection, the levels of $\mathrm{OH}^{-}$and $\mathrm{ONOO}^{-}$were significantly increased in oxaliplatin-injected mice $\left(\mathrm{P}<0.05, \mathrm{H}_{2} \mathrm{O}+\right.$ OXA vs $\mathrm{H}_{2} \mathrm{O}+$ Saline; $\mathrm{P}<0.05$, HW + OXA vs HW + Saline). As compared with

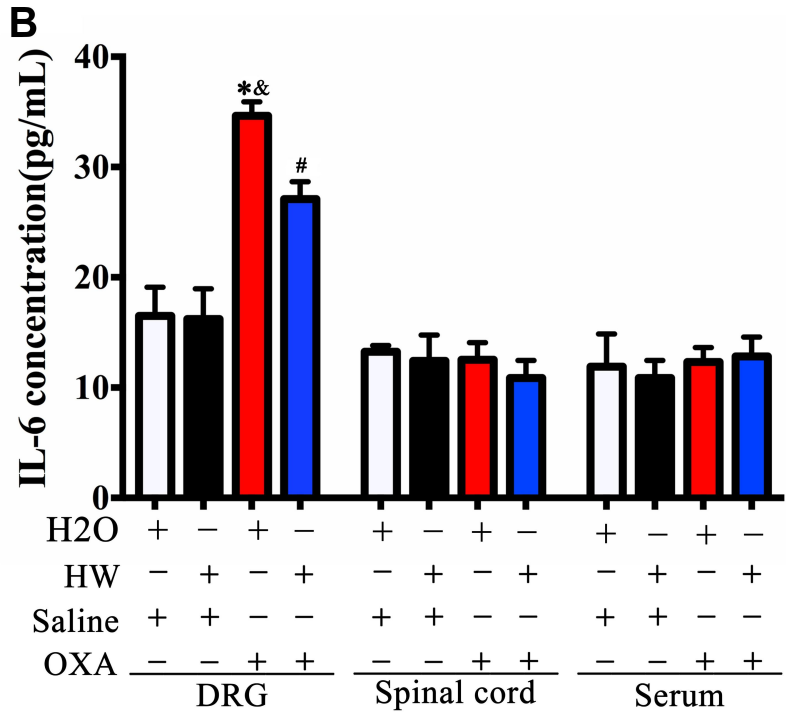

D

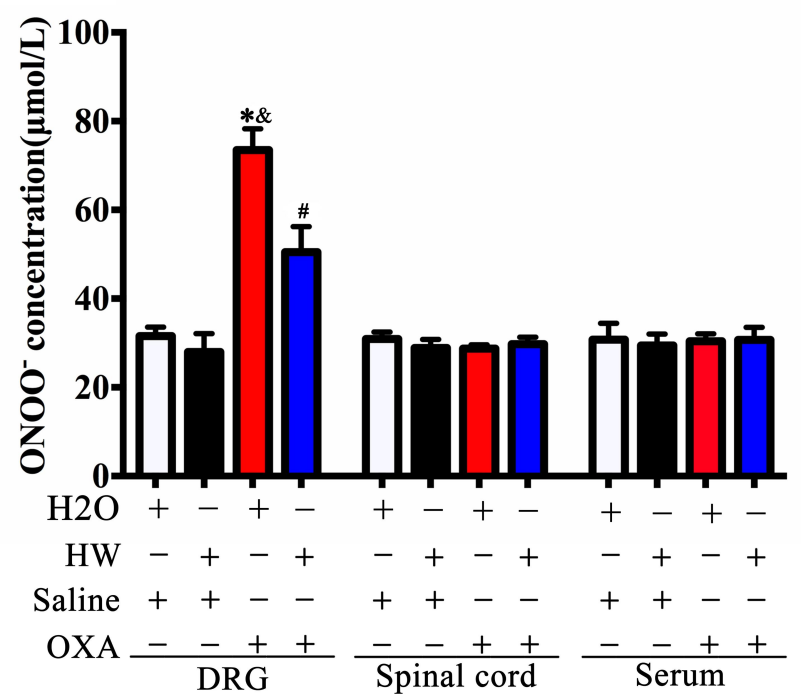

Figure 6 Effects of hydrogen-rich water on the expression of inflammatory cytokines and oxidative stress indicators in the DRG, spinal cords and serum of CINP mice. The pro-inflammatory cytokines (A) TNF- $\alpha$ and (B) IL-6, and oxidative stress indicators (C) $\mathrm{OH}^{-}$and (D) ONOO were measured in DRG, spinal cords and serum of mice by ELISA in each group ( $n=5$ mice per group). ${ }^{*} \mathrm{P}<0.05$ vs $\mathrm{H}_{2} \mathrm{O}+$ Saline group; ${ }^{\circledR} \mathrm{P}<0.05$ vs $\mathrm{HW}+$ Saline group; ${ }^{\#} \mathrm{P}<0.05$ vs $\mathrm{H}_{2} \mathrm{O}+\mathrm{OXA}$ group. Abbreviations: CINP, chemotherapy-induced neuropathic pain; DRG, dorsal root ganglia. 
the $\mathrm{H}_{2} \mathrm{O}+\mathrm{OXA}$ group, the levels of $\mathrm{OH}^{-}$and $\mathrm{ONOO}^{-}$in mice from the $\mathrm{HW}+\mathrm{OXA}$ group were significantly reduced $\left(\mathrm{P}<0.05\right.$, HW + OXA vs $\mathrm{H}_{2} \mathrm{O}+$ OXA). Similarly, the differences in the expression of $\mathrm{OH}^{-}$and $\mathrm{ONOO}^{-}$were only observed in DRG, and there were no significant differences in the spinal cords and serum (Figure 6C and D). These data indicated that DRG may play a key role in the pathogenesis of CINP, that oxaliplatin-induced hyperalgesia was associated with oxidative stress and inflammation, and that hydrogen-rich water could reverse this imbalance.

\section{Hydrogen-Rich Water Attenuates Oxaliplatin-Induced Hyperalgesia Caused by Gut Microbiota Through the LPS-TLR4} Pathway

Oxaliplatin-induced hyperalgesia was found to be associated with inflammatory response and gut microbiota; we therefore hypothesized that it may be mediated by LPS in the gut microbiota. The normal mice and mice drinking hydrogen-rich water were injected with oxaliplatin or saline. As shown in Figure 6A, oxaliplatin treatment could increase LPS levels in the serum and DRG of mice $\left(\mathrm{P}<0.05, \mathrm{H}_{2} \mathrm{O}+\right.$ OXA vs $\mathrm{H}_{2} \mathrm{O}+$ Saline; $\mathrm{P}<0.05, \mathrm{HW}+$ OXA vs HW + Saline), as compared with saline treatment, but no significant difference was observed in the spinal cords $(\mathrm{P}>0.05)$. At the same time, the levels of LPS in the serum and DRG of CINP mice drinking normal water were significantly higher than those of CINP mice drinking hydrogen-rich water $\left(\mathrm{P}<0.05, \mathrm{HW}+\mathrm{OXA}\right.$ vs $\mathrm{H}_{2} \mathrm{O}+$ OXA). Since LPS can be specifically recognized by TLR4, the expression of TLR4 was further detected. The Western blot analysis and ELISA results were consistent. As with LPS, TLR4 levels in the serum and DRG of oxaliplatintreated mice were markedly increased $\left(\mathrm{P}<0.05, \mathrm{H}_{2} \mathrm{O}+\right.$ OXA vs $\mathrm{H}_{2} \mathrm{O}+$ Saline; $\mathrm{P}<0.05$, HW + OXA vs $\mathrm{HW}+$ Saline), but the same was not observed in spinal cords $(\mathrm{P}>0.05)$; hydrogen-rich water could reduce this increase $\left(\mathrm{P}<0.05\right.$, HW + OXA vs $\mathrm{H}_{2} \mathrm{O}+$ OXA; Figure 7B-D). These results indicated that hydrogen-rich water could affect the gut microbiota and further reduce the occurrence of oxaliplatin-induced mechanical hyperalgesia through the LPS-TLR4 pathway.

\section{Discussion}

$\mathrm{H}_{2}$ is currently considered to be a promising medical gas with a potential to prevent or cure a range of diseases, such as ischemia-reperfusion injury, neurodegeneration, metabolic syndrome, inflammation, mitochondrial diseases, and even cancer. ${ }^{10}$ Previous studies published by our group have shown that hydrogen-rich water is resistant to certain diseases, and different methods of administering $\mathrm{H}_{2}$ are effective for the treatment of several intestinal diseases. ${ }^{12,26}$ The present study demonstrated that hydrogen-rich water has an effect on the diversity and structure of intestinal microbes, which may explain how hydrogenrich water can alleviate oxaliplatin-induced hyperalgesia.

In a previous study, a mouse CINP model was established by intraperitoneal injection of oxaliplatin, as previously described. ${ }^{5}$ The present study revealed that there was no significant difference in the mechanical threshold baseline between the different groups of mice. As compared to the baseline and mice in $\mathrm{H}_{2} \mathrm{O}+$ Saline, significant differences were observed in the mechanical threshold in the $\mathrm{H}_{2} \mathrm{O}+$ OXA group 5 days after oxaliplatin injection, with hyperalgesia occurring at 10 days in mice in the HW + OXA group. The behavioral results indicated that CINP mice exhibited pronounced hyperalgesia, while mechanical hyperalgesia in mice drinking hydrogen-rich water was significantly improved (Figure 3). In the present study, a mouse model of oxaliplatin-induced CINP was successfully established, and it was behaviorally proven that hydrogen-rich water could significantly improve mechanical hyperalgesia in mice with oxaliplatin-induced CINP.

The microbiota plays a pivotal role in the metabolism of its host. ${ }^{6}$ Under normal conditions, the intestinal resident bacteria and the host's micro-space structure form an interdependent and interacting micro-ecological system, and the intestinal micro-organism constitutes an important protective barrier against pathogens. ${ }^{5,27}$ When the stability of the micro-ecological microbiota is destroyed, the intestinal colonization resistance is greatly reduced, which can lead to the colonization and invasion of potential pathogens (including conditional pathogens) in the gut. ${ }^{23,24}$ In the present study, while oxaliplatin was injected, the mice were exposed to normal drinking water or hydrogen-rich water treatments, which were continued throughout the experiment. Good's coverage showed that the sample results could represent the true situation of microorganisms in mouse feces. It has been proven that changes in the gut microbiota do not change the tissue distribution of oxaliplatin, and studies have shown that the gut microbiota could alleviate oxaliplatin-induced pain. ${ }^{1,5,28}$ Consistent with previous studies, it was found herein that the 
A

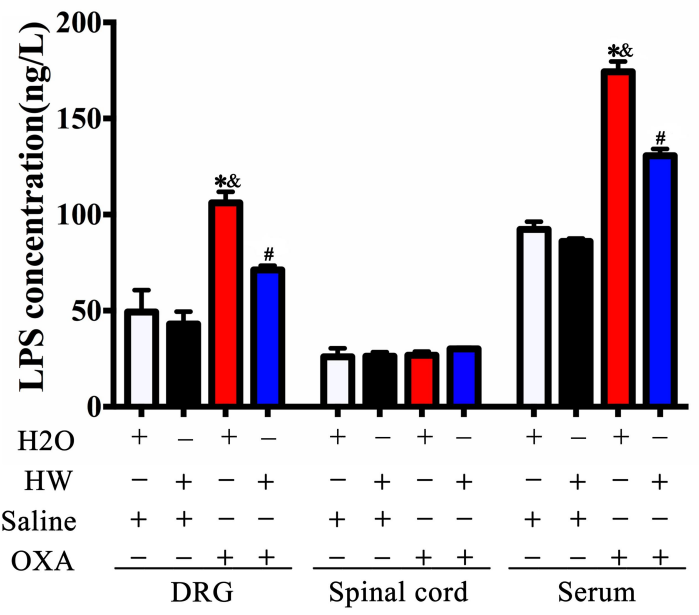

C

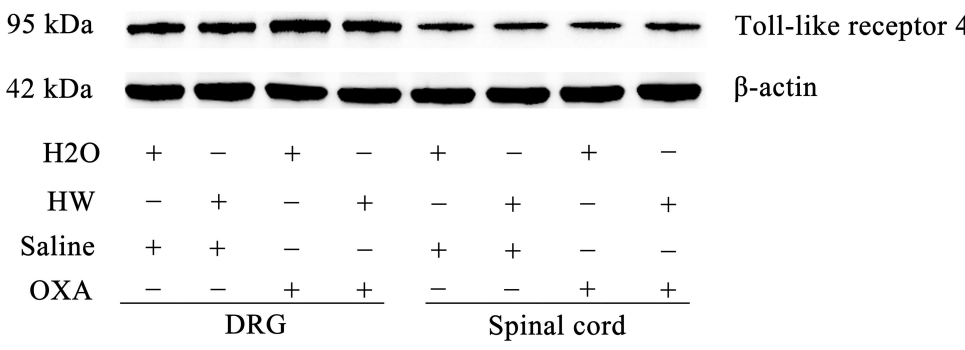

B

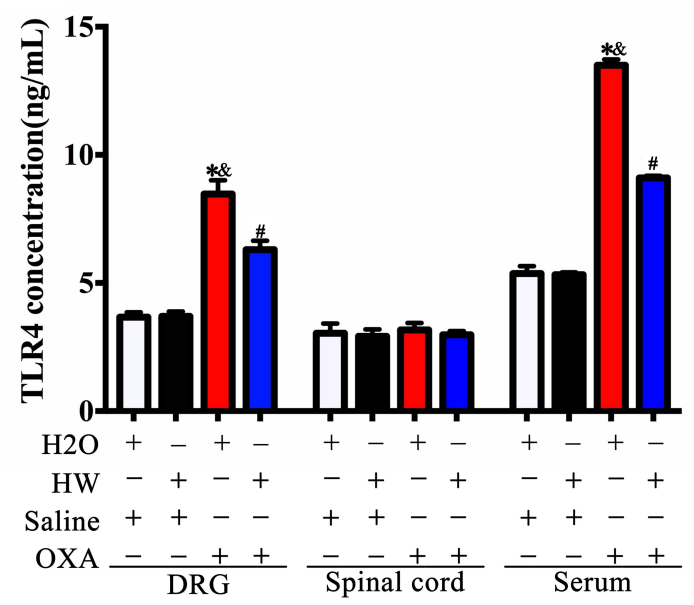

D

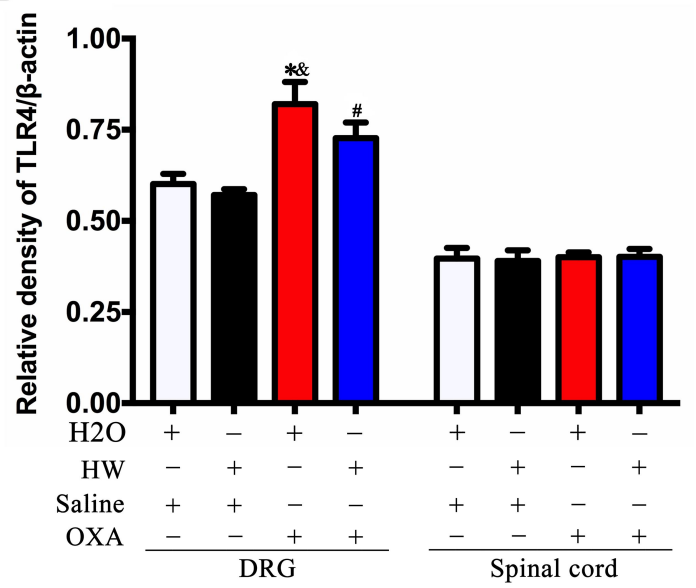

Figure 7 Effects of hydrogen-rich water on the expression of LPS and TLR4 in CINP mice. (A) The expression of LPS was detected in the DRG, spinal cords and serum of mice by ELISA in each group. (B) The expression of TLR4 was detected in the DRG, spinal cords and serum of mice by ELISA in each group. (C) The expression of TLR4 was detected by Western blot analysis. (D) Quantitative analysis of TLR4 is presented as the ratio of band density to that of $\beta$-actin. $n=5$ mice per group. $* P<0.05$ vs $\mathrm{H}_{2} \mathrm{O}+\mathrm{Saline}$ group; ${ }^{\circledR} \mathrm{P}<0.05$ vs $\mathrm{HW}+$ Saline group; ${ }^{\#} \mathrm{P}<0.05$ vs $\mathrm{H}_{2} \mathrm{O}+$ OXA group.

Abbreviations: LPS, lipopolysaccharide; TLR4, Toll-like receptor 4; CINP, chemotherapy-induced neuropathic pain; DRG, dorsal root ganglia.

composition and structure of gut microbiota of mice drinking the same water were changed following oxaliplatin treatment, indicating an association between intestinal microbiota and oxaliplatin. It was also demonstrated that hydrogen-rich water reduced the a-diversity measured by the Shannon and Simpson indices, and altered the community composition and structure of the intestinal microbiota (Figure 4). Shannon and Simpson indices are commonly used to estimate the diversity of microbiota in samples and reflect the a-diversity index. These two indices have opposite trends in their association with diversity; the higher the Shannon value, the higher the community diversity, and the higher the Simpson index, the lower the community diversity. It has been reported that hydrogen-rich water can significantly improve the gastrointestinal function and epithelial integrity of mice following total abdominal irradiation. ${ }^{29}$ Therefore, hydrogen-rich water can be considered to protect gastrointestinal function and epithelial integrity by altering the diversity and structure of mouse gut microbiota, thereby improving CINP.

Oxidative stress and excessive release of inflammatory cytokines are considered to be important factors of oxaliplatin-induced CINP. ${ }^{30}$ To evaluate the effect of hydrogenrich water on oxaliplatin-induced CINP, the expression of inflammatory cytokines was examined, including that of pro-inflammatory factors TNF- $\alpha$ and IL-6. The levels of oxidative stress indicators $\mathrm{OH}^{-}$and $\mathrm{ONOO}^{-}$were also examined. Oxidative stress is caused by the insufficient activity of the endogenous antioxidant defense system against reactive oxygen species, ${ }^{31}$ namely inflammatory response and oxidative stress imbalance. The results of the present study showed that oxaliplatin could 
significantly increase the levels of inflammatory cytokines TNF- $\alpha$ and IL- 6 in CINP mice, as well as the expression of oxidative stress indicators $\mathrm{OH}^{-}$and $\mathrm{ONOO}^{-}$, while hydrogen-rich water inhibited these increases. Among DRG, spinal cords and serum, these differences were only observed in DRG (Figure 6). The above findings supported the view that DRG may play a key role in the pathogenesis of CINP, that hyperalgesia caused by oxaliplatin is associated with excessive release of inflammatory cytokines and oxidative stress, and that hydrogen-rich water can reverse this imbalance.

It has been reported that the gut microbiota plays a key role in mediating inflammatory pain. ${ }^{5}$ The present findings suggested that gut microbiota, inflammatory responses and oxidative stress participate in oxaliplatin-induced mechanical hyperalgesia, and it was previously demonstrated that LPS is one of the key factors determining autoimmunity and inflammation derived from the gut microbiota. ${ }^{32}$ It was therefore hypothesized that oxaliplatin-induced hyperalgesia was mediated by gut microbiota-derived LPS. The present data indicated that oxaliplatin injection could increase the levels of LPS in the serum and DRG of mice, which was decreased following treatment with hydrogen-rich water; however, these differences were not reflected in the spinal cords. Since TLR4 is a ligand of LPS, ${ }^{33}$ TLR4 was further examined, and the result was the same as that for LPS (Figure 7). It can therefore be inferred that hydrogen-rich water affects the gut microbiota and reduces the occurrence of oxaliplatin-induced CINP through the LPS-TLR4 pathway.

However, this study was not without its limitations. First, it has been proved that altering the gut microbiota might alleviate oxaliplatin-induced pain in some studies. However, we only used hydrogen-rich water to treat CINP mice and find it could reverse the pain response and alter the microbiota in CINP mice. But we did not apply the fecal transplants to the CINP mice to see if it could attenuate or reverse the pain response under the condition of hydrogen-rich water drinking. We will confirm these in the future's research. Second, we only used female mice in the study, because females were not as aggressive as males, which were easier to do the behavioral test. And studies have already approved that there are no significant difference in oxaliplatin-induced CINP between male and female rodents. ${ }^{34,35}$

\section{Conclusions}

In conclusion, it was demonstrated that hydrogen-rich water affects the LPS-TLR4 pathway by altering the diversity and structure of the gut microbiota, thereby improving oxaliplatin-induced mechanical hyperalgesia and reversing the imbalance of inflammatory cytokines and oxidative stress, thus alleviating CINP. Therefore, $\mathrm{H}_{2}$ may be a promising method for alleviating CINP.

\section{Acknowledgments}

The present study was supported by the National Natural Science Foundation of China (grant nos. 82072150 and 82001149).

\section{Disclosure}

The authors report no conflicts of interest in this work.

\section{References}

1. Rossignol J, Cozzi B, Liebaert F, et al. High concentration of topical amitriptyline for treating chemotherapy-induced neuropathies. Support Care Cancer. 2019;27(8):3053-3059. doi:10.1007/s00520018-4618-y

2. Nakagawa T, Kaneko S. Roles of transient receptor potential ankyrin 1 in oxaliplatin-induced peripheral neuropathy. Biol Pharm Bull. 2017;40(7):947-953. doi:10.1248/bpb.b17-00243

3. Marmiroli P, Scuteri A, Cornblath DR, Cavaletti G. Pain in chemotherapy-induced peripheral neurotoxicity. $J$ Peripher Nerv Syst. 2017;22(3):156-161. doi:10.1111/jns.12226

4. Boyette-Davis JA, Hou S, Abdi S, Dougherty PM. An updated understanding of the mechanisms involved in chemotherapy-induced neuropathy. Pain Manag. 2018;8(5):363-375. doi:10.2217/pmt-20180020

5. Shen S, Lim G, You Z, et al. Gut microbiota is critical for the induction of chemotherapy-induced pain. Nat Neurosci. 2017;20 (9):1213-1216. doi:10.1038/nn.4606

6. Zhang H, Sparks JB, Karyala SV, Settlage R, Luo XM. Host adaptive immunity alters gut microbiota. ISME J. 2015;9(3):770-781. doi:10. 1038/ismej.2014.165

7. Marchesi JR, Adams DH, Fava F, et al. The gut microbiota and host health: a new clinical frontier. Gut. 2016;65(2):330-339. doi:10.1136/ gutjnl-2015-309990

8. Cheng S, Ma X, Geng S, et al. Fecal microbiota transplantation beneficially regulates intestinal mucosal autophagy and alleviates gut barrier injury. mSystems. 2018;3(5):5. doi:10.1128/mSystems.00137-18

9. Zhang C, Li S, Yang L, et al. Structural modulation of gut microbiota in life-long calorie-restricted mice. Nat Commun. 2013;4(1):2163. doi: $10.1038 /$ ncomms 3163

10. Xie K, Liu L, Yu Y, Wang G. Hydrogen gas presents a promising therapeutic strategy for sepsis. Biomed Res Int. 2014;2014:807635. doi:10.1155/2014/807635

11. Zhuang X, Yu Y, Jiang Y, et al. Molecular hydrogen attenuates sepsis-induced neuroinflammation through regulation of microglia polarization through an mTOR-autophagy-dependent pathway. Int Immunopharmacol. 2020;81:106287. doi:10.1016/j.intimp.2020. 106287

12. Yoneda T, Tomofuji T, Kunitomo M, et al. Preventive Effects of drinking hydrogen-rich water on gingival oxidative stress and alveolar bone resorption in rats fed a high-fat diet. Nutrients. 2017;9(1):1. doi:10.3390/nu9010064

13. Kasuyama K, Tomofuji T, Ekuni D, et al. Hydrogen-rich water attenuates experimental periodontitis in a rat model. $J$ Clin Periodontol. 2011;38(12):1085-1090. doi:10.1111/j.1600-051X.20 11.01801.x 
14. Yu Y, Yang Y, Yang M, Wang C, Xie K, Yu Y. Hydrogen gas reduces HMGB1 release in lung tissues of septic mice in an $\mathrm{Nrf2}$ HO-1-dependent pathway. Int Immunopharmacol. 2019;69:11-18. doi:10.1016/j.intimp.2019.01.022

15. Yu Y, Feng J, Lian N, et al. Hydrogen gas alleviates blood-brain barrier impairment and cognitive dysfunction of septic mice in an Nrf2-dependent pathway. Int Immunopharmacol. 2020;85:106585. doi:10.1016/j.intimp.2020.106585

16. Yan M, Yu Y, Mao X, et al. Hydrogen gas inhalation attenuates sepsis-induced liver injury in a FUNDC1-dependent manner. Int Immunopharmacol. 2019;71:61-67. doi:10.1016/j.intimp.2019. 03.021

17. Jiao Y, Yu Y, Li B, et al. Protective effects of hydrogen-rich saline against experimental diabetic peripheral neuropathy via activation of the mitochondrial ATP-sensitive potassium channel channels in rats. Mol Med Rep. 2020;21(1):282-290. doi:10.3892/mmr.2019.10795

18. Carchman EH, Whelan S, Loughran $P$, et al. Experimental sepsis-induced mitochondrial biogenesis is dependent on autophagy, TLR4, and TLR9 signaling in liver. FASEB J. 2013;27(12):47 03-4711. doi:10.1096/fj.13-229476

19. Li D, Gu R, Yang X, et al. TLR3 correlated with cervical lymph node metastasis in patients with papillary thyroid cancer. Int $J$ Clin Exp Med. 2014;7(12):5111-5117.

20. McCarron EP, Williams DP, Antoine DJ, et al. Exploring the translational disconnect between the murine and human inflammatory response: analysis of LPS dose-response relationship in murine versus human cell lines and implications for translation into murine models of sepsis. J Inflamm Res. 2015;8:201-209. doi:10.2147/JIR.S89097

21. Wang X, Li X, Zhang X, et al. Toll-like receptor 4-induced inflammatory responses contribute to the tumor-associated macrophages formation and infiltration in patients with diffuse large B-cell lymphoma. Ann Diagn Pathol. 2015;19(4):232-238. doi:10.1016/j. anndiagpath.2015.04.008

22. Yang J, Yuan F, Ye G, et al. Skin/Muscle incision and retraction induces evoked and spontaneous pain in mice. Pain Res Manag. 2019;2019:6528528. doi:10.1155/2019/6528528

23. Flores R, Shi J, Yu G, et al. Collection media and delayed freezing effects on microbial composition of human stool. Microbiome. 2015;3(1):33. doi:10.1186/s40168-015-0092-7

24. Plumb RS, Granger JH, Stumpf CL, et al. A rapid screening approach to metabonomics using UPLC and oa-TOF mass spectrometry: application to age, gender and diurnal variation in normal/Zucker obese rats and black, white and nude mice. Analyst. 2005;130(6):844-849. doi:10.1039/b501767j
25. Benton HP, Ivanisevic J, Mahieu NG, et al. Autonomous metabolomics for rapid metabolite identification in global profiling. Anal Chem. 2015;87(2):884-891. doi:10.1021/ac5025649

26. Yamasaki M, Miyazono M, Yoshihara M, et al. Effects of hydrogen-rich water in a rat model of polycystic kidney disease. PLoS One. 2019;14(4):e0215766. doi:10.1371/journal.pone.0215766

27. Finnicum CT, Beck JJ, Dolan CV, et al. Cohabitation is associated with a greater resemblance in gut microbiota which can impact cardiometabolic and inflammatory risk. BMC Microbiol. 2019;19 (1):230. doi:10.1186/s12866-019-1602-8

28. Pulvers JN, Marx G. Factors associated with the development and severity of oxaliplatin-induced peripheral neuropathy: a systematic review. Asia Pac J Clin Oncol. 2017;13(6):345-355. doi:10.1111/ ajco. 12694

29. Xiao H-W, Li Y, Luo D, et al. Hydrogen-water ameliorates radiationinduced gastrointestinal toxicity via MyD88's effects on the gut microbiota. Exp Mol Med. 2018;50(1):e433. doi:10.1038/emm.20 17.246

30. Duan Z, Su Z, Wang H, Pang X. Involvement of pro-inflammation signal pathway in inhibitory effects of rapamycin on oxaliplatin-induced neuropathic pain. Mol Pain. 2018;14:1744806918769426. doi:10.1177/ 1744806918769426

31. Miletic J, Drakulic D, Pejic S, et al. Prooxidant-antioxidant balance, advanced oxidation protein products and lipid peroxidation in Serbian patients with Parkinson's disease. Int $J$ Neurosci. 2018;128 (7):600-607. doi:10.1080/00207454.2017.1403916

32. Vatanen T, Kostic AD, d'Hennezel E, et al. Variation in Microbiome LPS Immunogenicity contributes to autoimmunity in humans. Cell. 2016;165(6):1551. doi:10.1016/j.cell.2016.05.056

33. Ding D, Xu S, Zhang H, et al. 3-Methyladenine and dexmedetomidine reverse lipopolysaccharide-induced acute lung injury through the inhibition of inflammation and autophagy. Exp Ther Med. 2018;15(4):3516-3522. doi:10.3892/etm.2018.5832

34. Brewer A, Shirachi D, Quock R, et al. Effect of hyperbaric oxygen on chemotherapy-induced neuropathy in male and female rats. Behav Pharmacol. 2020;31(1):61-72. doi:10.1097/FBP.0000000000000497

35. Legakis L, Diester C, Townsend E, et al. Comparison of chemotherapy effects on mechanical sensitivity and food-maintained operant responding in male and female rats. Behav Pharmacol. 2020;31 (5):477-490. doi:10.1097/FBP.000000000000052
Journal of Pain Research

\section{Publish your work in this journal}

The Journal of Pain Research is an international, peer reviewed, open access, online journal that welcomes laboratory and clinical findings in the fields of pain research and the prevention and management of pain. Original research, reviews, symposium reports, hypothesis formation and commentaries are all considered for publication. The manuscript

Submit your manuscript here: https://www.dovepress.com/journal-of-pain-research-journa management system is completely online and includes a very quick and fair peer-review system, which is all easy to use. Visit http:// www.dovepress.com/testimonials.php to read real quotes from published authors. 